\title{
Quantitative theory of triplet pairing in the unconventional superconductor $\mathrm{LaNiGa}_{2}$
}

\author{
Sudeep Kumar Ghosh $\odot,{ }^{1, *}$ Gábor Csire, ${ }^{2,3, \dagger}$ Philip Whittlesea, ${ }^{1}$ James F. Annett, ${ }^{2}$ Martin Gradhand, ${ }^{2}$ \\ Balázs Újfalussy $\odot,{ }^{4}$ and Jorge Quintanilla ${ }^{1, \ddagger}$ \\ ${ }^{1}$ Physics of Quantum Materials, School of Physical Sciences, University of Kent, Canterbury CT2 7NH, United Kingdom \\ ${ }^{2}$ H. H. Wills Physics Laboratory, University of Bristol, Tyndall Avenue, Bristol BS8 1TL, United Kingdom \\ ${ }^{3}$ Catalan Institute of Nanoscience and Nanotechnology (ICN2), CSIC, BIST, Campus UAB, Bellaterra, Barcelona, 08193, Spain \\ ${ }^{4}$ Institute for Solid State Physics and Optics, Wigner Research Centre for Physics, Hungarian Academy of Sciences, \\ P.O. Box 49, H-1525 Budapest, Hungary
}

(Received 24 December 2019; revised manuscript received 13 March 2020; accepted 16 March 2020; published 30 March 2020)

\begin{abstract}
The exceptionally low-symmetry crystal structures of the time-reversal symmetry-breaking superconductors $\mathrm{LaNiC}_{2}$ and $\mathrm{LaNiGa}_{2}$ lead to an internally antisymmetric nonunitary triplet state as the only possibility compatible with experiments. We argue that this state has a distinct signature: a double-peak structure in the density of states (DOS) which resolves in the spin channel in a particular way. We construct a detailed model of $\mathrm{LaNiGa}_{2}$ capturing its electronic band structure and magnetic properties ab initio. The pairing mechanism is described via a single adjustable parameter. The latter is fixed by the critical temperature $T_{c}$ allowing parameter-free predictions. We compute the electronic specific heat and find excellent agreement with experiment. The size of the ordered moment in the superconducting state is compatible with zero-field muon spin relaxation experiments and the predicted spin-resolved DOS suggests the spin splitting is within the reach of present experimental technology.
\end{abstract}

DOI: 10.1103/PhysRevB.101.100506

The superconducting state is a condensate of electron pairs characterized by an order parameter $\Delta$. Usually $\Delta$ is a complex scalar, its phase being a manifestation of spontaneously broken gauge symmetry. This is responsible for the macroscopic quantum coherence underpinning quantum devices such as superconducting qubits [1] and superconducting quantum interference devices (SQUIDs) [2]. On the other hand, in so-called "unconventional" superconductors additional symmetries may be broken leading to more complex order parameters with extra degrees of freedom. Of all the features of unconventional superconductors, broken time-reversal symmetry (TRS) is perhaps the most surprising one as it challenges our view of superconductivity and magnetism as antagonistic states of matter. In spite of this, the phenomenon has been detected in numerous systems using zero-field muon spin rotation/relaxation $(\mu \mathrm{SR})$. Prominent examples include (U, Th) $\mathrm{Be}_{13}[3], \mathrm{Sr}_{2} \mathrm{RuO}_{4}$ [4], $\mathrm{UPt}_{3}$ [5], $(\mathrm{Pr}, \mathrm{La})(\mathrm{Ru}, \mathrm{Os})_{4} \mathrm{Sb}_{12}$ [6,7], $\mathrm{PrPt}_{4} \mathrm{Ge}_{12}$ [8], $\mathrm{LaNiC}_{2}$ [9], $\mathrm{LaNiGa}_{2}$ [10,11], SrPtAs [12], $\mathrm{Re}_{6}(\mathrm{Zr}, \mathrm{Hf}, \mathrm{Ti})$ [13-16], $\mathrm{Lu}_{5} \mathrm{Rh}_{6} \mathrm{Sn}_{18}$ [17], and $\mathrm{La}_{7}(\mathrm{Ir}, \mathrm{Rh})_{3}$ [18,19]. Many of these systems have other unconventional features, while in some cases an independent, direct observation of broken TRS has been made: optical Kerr effect measurement in $\mathrm{Sr}_{2} \mathrm{RuO}_{4}$ [20] and $\mathrm{UPt}_{3}$ [21], and bulk SQUID magnetization measurement in $\mathrm{LaNiC}_{2}$ [22].

\footnotetext{
*S.Ghosh@kent.ac.uk

†gabor.csire@icn2.cat

¥J.Quintanilla@kent.ac.uk
}

Unfortunately it has been difficult to establish the structures of order parameters of these superconductors. This is because, on the one hand, our knowledge of the electron pairing mechanism is not sufficiently detailed to make a prediction. On the other hand, their crystal structures tend to be highly symmetric, leading to many different possible ways of breaking TRS, which limits our ability to work by elimination. TRS-breaking superconductivity requires a degenerate instability channel $[23,24]$ which, for a uniform superconductor, must come from a multidimensional irreducible representation (irrep) of the point group of the crystal. As an example, the point group of $\mathrm{Sr}_{2} \mathrm{RuO}_{4}$ is $D_{4 h}$, which leads to 22 possible order parameters breaking TRS [23,24]: 20 under the assumption of weak spin-orbit coupling (SOC) and two more in the strong-SOC limit. The family formed by $\mathrm{LaNiC}_{2}$ [9] and $\mathrm{LaNiGa}_{2}$ [10] is an exception to this rule, as their crystal structures have exceptionally low symmetry. Their crystal point groups only have four irreps, all of them one dimensional. This precludes TRS breaking in the strongSOC case and leaves only four possible pairing states, all of them nonunitary triplets $[10,25]$. One additional complication is the multiband nature of these systems: two [26] and five [27] bands cross the Fermi level of $\mathrm{LaNiC}_{2}$ and $\mathrm{LaNiGa}_{2}$, respectively. In fact, both systems show thermodynamic properties that can be fitted with a model assuming fully gapped, two-band superconductivity $[11,28,29]$. This is inconsistent with the line nodes implied by the earlier symmetry analyses $[10,25]$. On the other hand, the two-band model does not predict TRS breaking. To resolve the discrepancy it was proposed that only an internally antisymmetric nonunitary triplet pairing (INT) state is compatible with the experimental 
observations $[10,11,25,30]$. Here we show that such a state has a very distinct experimental signature: a double-peak structure in the density of states (DOS) which resolves in the spin channel. We construct a model of $\mathrm{LaNiGa}_{2}$ capturing detailed electronic band structure $a b$ initio, with the pairing interaction in the INT state reduced to a single, adjustable parameter. The known value of the critical temperature $T_{c}$ fixes this single parameter, allowing us to make parameter-free predictions. We obtain the electronic specific heat and find an excellent agreement with experiment [11]. We compute the spin-resolved DOS having a double-peak structure with each peak corresponding to a single spin channel. We find that the splitting is $\sim 0.2 \mathrm{meV}$-within the reach of present experimental technology.

The triplet pairing in the INT state relies heavily on the interband pairing, which enables an isotropic gap function and equal-spin pairing breaking TRS [11]. The Cooper pair wave function is symmetric in the crystal momentum and spin channels but it is antisymmetric with respect to the orbital degree of freedom. Recent studies [11,31-42] in several materials, including the iron-based superconductors, half-Heusler compounds, $\mathrm{UPt}_{3}$ and $\mathrm{Sr}_{2} \mathrm{RuO}_{4}$, have also pointed out the importance of internal degrees of freedom of electrons (coming from, e.g., sublattice or multiple orbitals) in determining the pairing symmetries of superconducting ground states.

A convenient toy model of low-energy excitations in the INT state proposed in Ref. [11] is provided by the following Bogoliubov-de Gennes (BdG) Hamiltonian:

$$
\mathcal{H}=\left(\begin{array}{cc}
\mathcal{H}_{0}(\boldsymbol{k}) & \hat{\Delta} \\
\hat{\Delta}^{\dagger} & -\mathcal{H}_{0}(\boldsymbol{k})
\end{array}\right) .
$$

Here $\mathbf{k}$ is the crystal momentum of the excitation,

$$
\mathcal{H}_{0}(\boldsymbol{k})=\mathbb{1}_{2} \otimes\left(\begin{array}{cc}
\epsilon_{0}(\boldsymbol{k})-\mu-s & \delta \\
\delta & \epsilon_{0}(\boldsymbol{k})-\mu+s
\end{array}\right)
$$

is the normal-state, single-electron Hamiltonian with the chemical potential $\mu$, and

$$
\hat{\Delta}=i(\boldsymbol{d} \cdot \boldsymbol{\sigma}) \sigma_{y} \otimes i \tau_{y}
$$

represents the pairing potential. In the tensor products, the first sector represents the spin channels $\sigma=\uparrow, \downarrow$ while the second represents the two orbital channels. For the purpose of initial discussion, we have assumed a very simple band structure with two bands labeled by $m=+$ and - , one emerging from each orbital, that are related by a rigid energy shift $2 s$ and with a $\mathbf{k}$-independent hybridization factor $\delta$. The pairing matrix describes k-independent triplet pairing but is antisymmetric in the orbital channel in order to ensure the fermionic antisymmetry of the Cooper pair wave function [11,31]. Here, $\sigma$ and $\tau$ are the vectors of Pauli matrices in the spin and orbital sectors, respectively. Writing the triplet $d$ vector in the form $\boldsymbol{d}=\Delta_{0} \boldsymbol{\eta}$, where $|\boldsymbol{\eta}|^{2}=1$ and $\Delta_{0}$ is a pairing amplitude, the nonunitarity of the triplet state is characterized by a nonzero real vector $\boldsymbol{q}=i\left(\boldsymbol{\eta} \times \boldsymbol{\eta}^{*}\right)$ which in general has $|\boldsymbol{q}| \leqslant|\boldsymbol{\eta}|^{2}=1$.

Diagonalizing $\mathcal{H}$ yields the quasiparticle spectrum $E_{k}$ shown, for a particular choice of parameters, in Fig. 1(a). The plot is representative of cases where $s, \delta \ll \Delta_{0}$. This is the physically relevant regime for the toy model as in a mean-field picture the pairing amplitude $\left(\Delta_{0}\right)$ has to be able to overcome
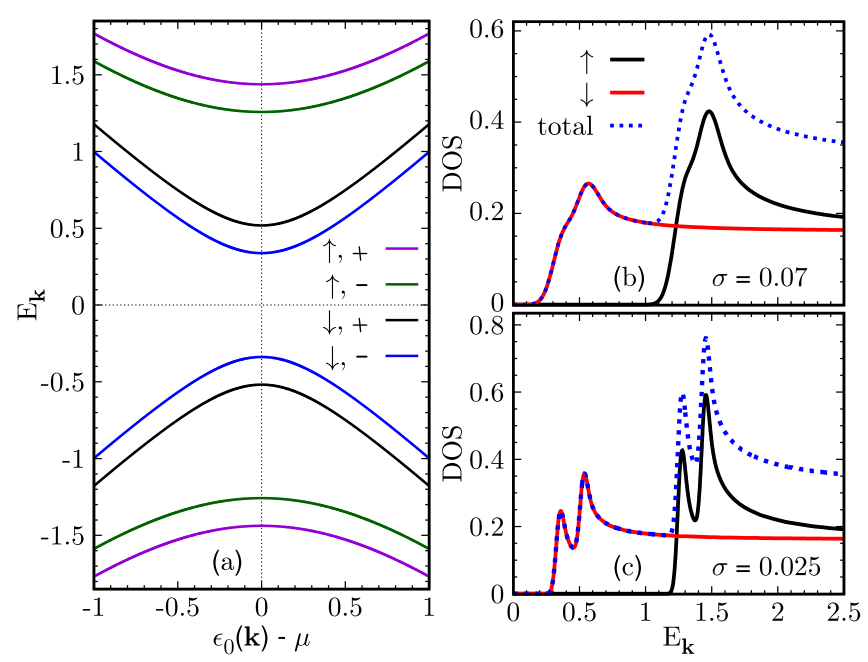

FIG. 1. Properties of quasiparticles in the INT state with $s=$ $0.05, \delta=0.075,\left|\Delta_{0}\right|=1$, and $|\boldsymbol{q}|=\sqrt{2 / 3}$ in arbitrary units. (a) Quasiparticle spectrum for the + and - bands for $\uparrow$ and $\downarrow$ spins. (b) and (c) show the corresponding DOS calculated from this spectrum using the same parameters. The DOS features have been artificially broadened by convolution with a Gaussian of width $\sigma=0.07$ in (b) and $\sigma=0.025$ in (c).

the band splitting $\sim \delta, s$. This unrealistic requirement is relaxed when the band splitting is allowed to be $k$ dependent, as in the more detailed model discussed below. As indicated in the plot, each excitation has well-defined band and spin indices. The Bogoliubov bands are paired up, with each member of the pair sharing the spin index but differing in the band index. The corresponding DOS is displayed in Figs. 1(b) and 1(c). Here we have introduced two different levels of broadening to simulate different experimental resolutions in the two figures. The DOS is fully gapped, with four pairs of coherence peaks that are grouped in two doublets, depending on the level of broadening. Crucially, the spin-resolved DOS shows only one of the two doublets in each spin channel. This qualitative feature distinguishes this double-peak structure from that which would be obtained, for example, in a multiband superconductor. The observation of such a spin-resolved feature would provide definitive proof of the INT state.

An analytical formula for $E_{k}$ can be easily obtained in the limits $s \rightarrow 0$ or $\delta \rightarrow 0$. In either case, the result is

$$
E_{k}= \pm\left[ \pm a+\sqrt{\left\{\epsilon_{0}(\boldsymbol{k})-\mu\right\}^{2}+\left|\Delta_{0}\right|^{2}(1 \pm|\boldsymbol{q}|)}\right]
$$

where $a=\delta$ or $s$, respectively. Note that both $\delta$ and $s$ play similar roles. This shows that our toy model does not rely on the two bands being orthogonal. The above formula can be used to estimate the ratio between the gaps in the energy spectrum for spin-up $\left(\mathcal{E}_{\uparrow \uparrow}\right)$ and spin-down $\left(\mathcal{E}_{\downarrow \downarrow}\right)$ quasiparticles. In the limit $a \ll \Delta_{0}$, it is

$$
\frac{\mathcal{E}_{\uparrow \uparrow}}{\mathcal{E}_{\downarrow \downarrow}}=\frac{\sqrt{1+|\boldsymbol{q}|}}{\sqrt{1-|\boldsymbol{q}|}}=1-|\boldsymbol{q}|+O\left(|\boldsymbol{q}|^{2}\right) .
$$

The above toy model assumes that an isotropic, equal-spin pairing potential can lower the free energy in spite of the need for it to breach the energy gap between the bands. We explicitly show this by considering the toy many-body 


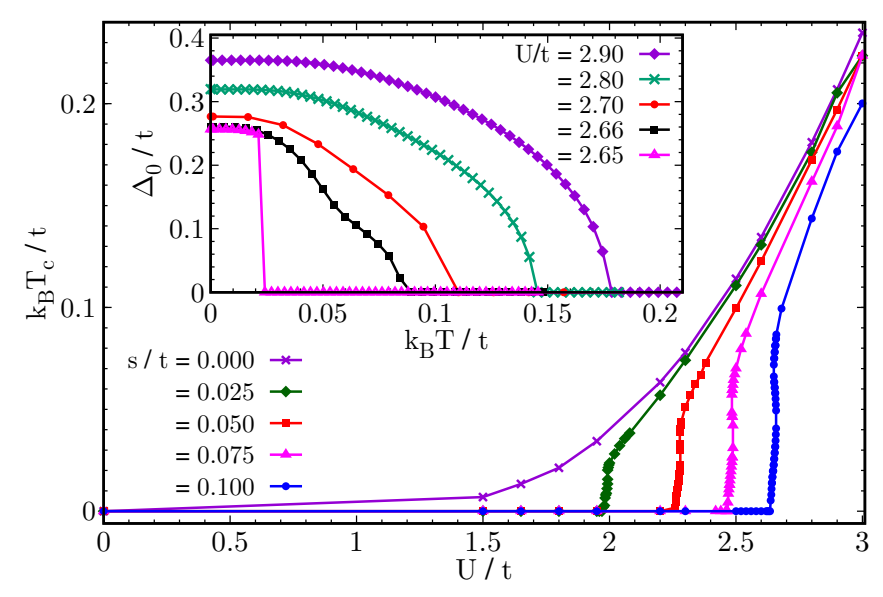

FIG. 2. Superconducting phase diagram of the toy model in Eq. (6). $T_{c}$ is the critical temperature where interorbital, equal-spin pairing sets in. Each curve shows the dependence of $T_{c}$ on the interaction strength $U$ for a different value of the band splitting $2 s$, as indicated. The inset shows the temperature dependence of the pairing amplitude $\Delta_{0}$ for the largest splitting $s / t=0.1$ and a few values of $U$ just above the critical value $U_{c}$ at which $T_{c}$ becomes finite.

Hamiltonian

$$
\hat{H}=\sum_{k} \hat{\Psi}_{k}^{\dagger} \mathcal{H}_{0}(\boldsymbol{k}) \hat{\Psi}_{k}+\hat{H}_{I}
$$

Here $\Psi_{\boldsymbol{k}}=\left(\hat{c}_{\boldsymbol{k},+, \uparrow}, \hat{c}_{\boldsymbol{k},+, \downarrow}, \hat{c}_{\boldsymbol{k},-, \uparrow}, \hat{c}_{\boldsymbol{k},-, \downarrow}\right)$, where $\hat{c}_{\boldsymbol{k}, m, \sigma}$ creates an electron in the $m$ th band with crystal momentum $\boldsymbol{k}$ and spin $\sigma$. The single-electron Hamiltonian $\mathcal{H}_{0}(\boldsymbol{k})$ is given in Eq. (2) where for simplicity we take $\delta=0$ and $\epsilon_{0}(\boldsymbol{k})=$ $-2 t\left[\cos \left(k_{x}\right)+\cos \left(k_{y}\right)\right]$. We consider an on-site, interorbital, equal-spin pairing interaction proposed in Ref. [11], which can be written [43] as

$$
\hat{H}_{I}=-U \sum_{\boldsymbol{k}, \boldsymbol{k}^{\prime}, \sigma} c_{\boldsymbol{k},+, \sigma}^{\dagger} c_{-\boldsymbol{k},-, \sigma}^{\dagger} c_{-\boldsymbol{k}^{\prime},-, \sigma} c_{\boldsymbol{k}^{\prime},+, \sigma}
$$

with $U>0$ being the effective attraction strength. A standard mean-field treatment of this model [44] yields the phase diagram shown in Fig. 2. In the limit $s \rightarrow 0$, the theory is formally equivalent to two copies of a BCS theory, but with the band index $m$ playing the role of the spin index (one copy corresponding to each value of the real spin). For finite $s$, a critical interaction strength $U_{c}$ is necessary for the critical temperature $T_{c}$ to be finite, but for $U \gg U_{c}$ the results are very similar to the case $s \rightarrow 0$. This is confirmed by the inset, showing the temperature dependence of the pairing amplitude $\Delta_{0}$. While the superconducting transition can be of first order and even reentrant (not shown; see Ref. [44]) for a very narrow window $U \gtrsim U_{c}$, and displays some shoulders for slightly larger $U$, BCS-like behavior is recovered for $U \gg U_{c}$.

The above simple calculation shows that an equal-spin pairing potential can, in principle, breach a band gap to lead to a fully gapped triplet pairing state. On the other hand, our simple mean-field theory yields $\boldsymbol{\eta}=(0,1,0)$, i.e., a unitary triplet pairing state with $\boldsymbol{q}=0$. A more realistic theory must treat the pairing and exchange fields on equal footing. Symmetry arguments [10] show that any triplet instability leads to a subdominant magnetization, which lowers the free energy of the nonunitary state with $|\mathbf{q}| \neq 0[10,45]$. We now therefore build a more sophisticated, realistic description which not only incorporates an accurate description of the exchange field, but also a realistic prediction of the normal-state electronic structure of $\mathrm{LaNiGa}_{2}$, making quantitative predictions of superconducting properties possible.

Density-functional theory (DFT) in the local-density approximation (LDA) shows that $\mathrm{LaNiGa}_{2}$ is a multiband superconductor with several bands crossing the Fermi level giving rise to multiple Fermi surface sheets [27]. None of the bands can be obtained from one another through a simple rigid shift as in our toy model. There are, however, several regions within the Brillouin zone where the pairs of Fermi surface sheets are parallel and very close to each other, that is, nearly degenerate [44]. Moreover, the five bands have mixed Ni $3 d$, La $5 d$, and Ga $4 p$ characters. As a result, the Fermi surfaces have strong orbital degeneracy. To capture these details, we adopt a semiphenomenological strategy [46]. We consider the relativistic version of the BdG Hamiltonian in Eq. (1) together with the realistic LDA band structure and a phenomenological pairing interaction of the type given by Eq. (7). This leads to the Kohn-Sham-Dirac-BdG Hamiltonian [47]

$$
\mathcal{H}_{\mathrm{DBdG}}=\left[\begin{array}{cc}
H_{D} & \hat{\Delta}(\boldsymbol{r}) \\
\hat{\Delta}^{\dagger}(\boldsymbol{r}) & -H_{D}^{*}
\end{array}\right],
$$

where $H_{D}$ is the effective normal state Dirac Hamiltonian given by

$$
\begin{aligned}
H_{D}= & c \boldsymbol{p} \hat{\boldsymbol{\alpha}}_{1}+\left(\hat{\boldsymbol{\alpha}}_{2}-\mathbb{1}_{4}\right) c^{2} / 2 \\
& +\left[V_{\mathrm{eff}}(\boldsymbol{r})-E_{F}\right] \mathbb{1}_{4}+\mathbf{B}_{\mathrm{eff}}(\boldsymbol{r}) \hat{\boldsymbol{\alpha}}_{3} .
\end{aligned}
$$

Here, $\hat{\boldsymbol{\alpha}}_{1}=\hat{\boldsymbol{\sigma}}_{x} \otimes \hat{\boldsymbol{\sigma}}, \hat{\boldsymbol{\alpha}}_{2}=\hat{\boldsymbol{\sigma}}_{z} \otimes \mathbb{1}_{2}$, and $\hat{\boldsymbol{\alpha}}_{3}=\mathbb{1}_{2} \otimes \hat{\boldsymbol{\sigma}}$ with $\hat{\boldsymbol{\sigma}}$ being the Pauli matrices and $\mathbb{1}_{n}$ being the identity matrix of order $n . V_{\text {eff }}(\boldsymbol{r})$ and $\mathbf{B}_{\text {eff }}(\boldsymbol{r})$ are the effective electrostatic potential and the effective exchange field, respectively. $\hat{\Delta}(\boldsymbol{r})$ is the $4 \times 4$ pairing potential matrix due to the four-component Dirac spinors. Requiring self-consistency in the electrostatic potential, exchange field, and pairing potential, the solution is provided by our recently developed method [47] which generalizes the Korringa-Kohn-Rostoker (KKR) formalism. Within the KKR formalism intraorbital and interorbital pairings could be described both in the singlet and triplet channel by transforming the $(L, \sigma)$ representation of the pairing potential into a relativistic basis set, where $L$ refers to the real spherical harmonics assuming that the $z$ direction is perpendicular to the layered structure of $\mathrm{LaNiGa}_{2}$. The technical details are given in Ref. [44].

It is important to note that the ground state does not show ferromagnetism in the normal state, although there is a significant contribution from the $\mathrm{Ni} 3 d$ states at the Fermi level [27]. Since it is known that Hund's rule coupling plays an important role on the $\mathrm{Ni}$ atoms $[48,49]$, and on the other hand Hund's coupling can also produce local pairing [50], it is physically reasonable to assume an interorbital equalspin pairing involving two orbitals on the same $\mathrm{Ni}$ atom. We describe this by a two-body on-site attractive interaction $U_{L, L^{\prime}}$ between electrons with equal spins in only two of these orbitals $\left(L \neq L^{\prime}\right)$ with the pairing potential satisfying the self-consistency equation: $\Delta_{L \sigma, L^{\prime} \sigma}(\boldsymbol{r})=U_{L, L^{\prime}} \chi_{L \sigma, L^{\prime} \sigma}(\boldsymbol{r})$ where $\chi_{L \sigma, L^{\prime} \sigma}(\boldsymbol{r})$ is the corresponding pairing amplitude. Since all 

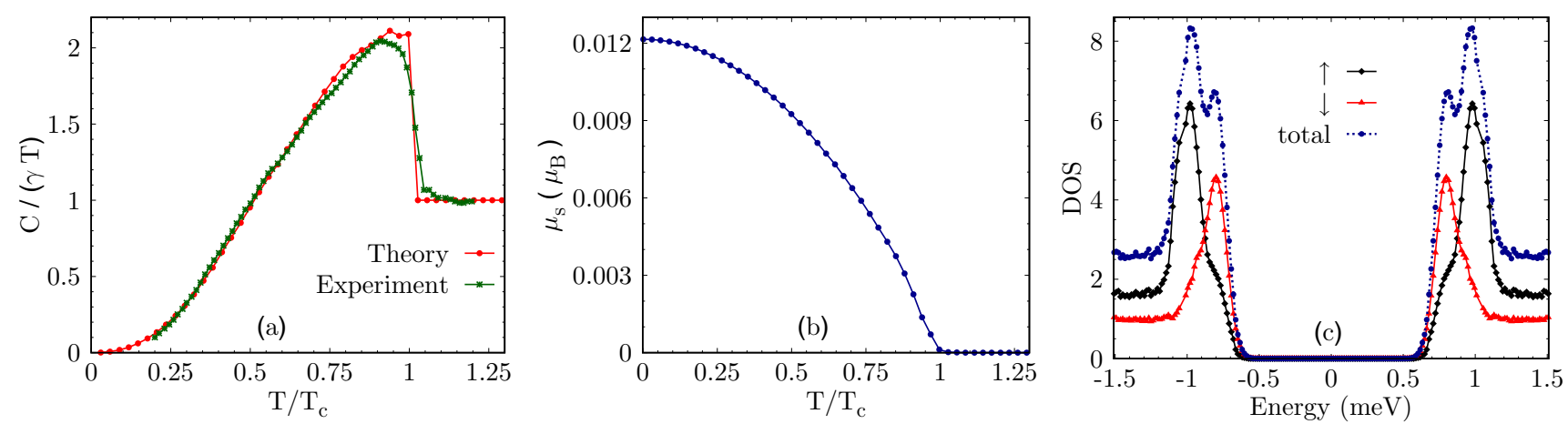

FIG. 3. Properties of the superconducting ground state corresponding to a phenomenological interorbital equal-spin pairing interaction between the $\left(d_{z^{2}}-d_{x y}\right)$ orbitals in the $3 d$ sector of the $\mathrm{Ni}$ atom in $\mathrm{LaNiGa}_{2}$. (a) Variation of the specific heat with temperature $(\gamma$ is the Sommerfeld coefficient). We note an excellent agreement between the theoretical result and the experimental data taken from Ref. [11]. (b) Variation of the spontaneous magnetic moment $\left(\mu_{s}\right)$ as a function of temperature. A clear increase in the magnetic moment below $T_{c}$ is an indication of the imbalance between two spin species due to migration of Cooper pairs. (c) Spin-resolved density of states (arbitrary units) of the Bogoliubov quasiparticles as a function of energy. We note that the two coherence peaks correspond to up and down species of Cooper pairs leading to two gaps in the quasiparticle spectrum.

of the Ni $d$ orbitals contribute to the density of states at the Fermi level, there are ten possible pairing models within this approach. Only one of the ten possible combinations, namely, pairing between $d_{z^{2}}$ and $d_{x y}$, yields a fully gapped quasiparticle spectrum (all the other possibilities have nodes on at least one of the Fermi surface sheets; see Ref. [44]). The strength $U_{d_{2}, d_{x y}}$ of the interaction between these two orbitals is the only adjustable parameter in our theory, to be fixed by requiring $T_{c}$ to be the same as in experiments [11]. Then we can make parameter-free predictions of observable properties of the system. The requirement that $T_{c}$ is the same as in experiments leads to $U_{d_{2}, d_{x y}}=0.65 \mathrm{eV}$, which is comparable to the values of Hund's coupling found for $\mathrm{Ni}$ atoms [49]. This result should motivate high-pressure measurements and dynamical mean-field theory studies to further explore the role of electronic correlations involving Hund's coupling. We stress that the present attractive interaction is described by a phenomenological parameter and therefore our calculation cannot directly address the question of its origin.

Having fixed our single parameter, we can now make parameter-free predictions. We first compute the specific heat of $\mathrm{LaNiGa}_{2}$ as a function of temperature by evaluating the temperature dependence of the quasiparticle DOS selfconsistently [46,51]. It is shown in Fig. 3(a) as compared to the corresponding experimental data from Ref. [11]. The agreement is excellent, suggesting that the observed twogap behavior of this curve is consistent with our equal-spin, interorbital pairing model.

The solution of the self-consistency equations reveals a charge imbalance between $\uparrow \uparrow(67 \%)$ and $\downarrow \downarrow$ (33\%) triplet components on the Ni atom. The migration of Cooper pairs from the minority $\downarrow \downarrow$ state to the majority $\uparrow \uparrow$ state is expected to generate a finite magnetization. Since our pairing interaction $U_{d_{z}, d_{x y}}$ is spin independent (hence preserves TRS), we have found a spontaneous TRS breaking in the INT state, which is a perfect analog of a ferromagnetic transition in a normal-state DFT calculation. The pairing-induced spontaneous magnetization $\left(\mu_{s}\right)$ is shown in Fig. 3(b). The magnetic moment is expected to vary linearly very close to
$T_{c}$ [10]; however, this behavior is hard to resolve here due to demanding numerical accuracy near $T_{c}$. We can estimate the size of the internal magnetic field at zero temperature using $\mu_{s}^{0}$, the value of $\mu_{s}$ at $T=0$, as $B_{\text {int }} \approx \frac{\mu_{0} \mu_{s}^{0}}{4 \pi a b c} \approx 0.3 \mathrm{G}$ which is of similar order as seen in the zero-field $\mu$ SR measurements [10].

Finally, we compute the spin-resolved quasiparticle DOS of $\mathrm{LaNiGa}_{2}$ as shown in Fig. 3(c). Its similarity with Fig. 1(b) is striking, confirming that our DFT-KKR calculation for this material describes the same physics. The two distinct superconducting gaps are clearly visible, and the spin-resolved curves show that they correspond to different spin species. The double-peak structure of the DOS is our main prediction. We note that the splitting between the two peaks is of the order of $0.2 \mathrm{meV}$-within the resolution of current scanning tunneling microscopy [52], photoemission [53], and tunneling experiments [54]. The crucial feature is that, unlike the case of a multiband superconductor [55], the two peaks correspond to distinct spin channels. Verifying this experimentally would thus require spin resolution [56].

Interestingly, in Ref. [11] the specific heat measurement was fitted by a phenomenological two-band model leading to the gap values $\Delta_{1}=1.08 k_{B} T_{c}$ and $\Delta_{2}=2.06 k_{B} T_{c}$, while we find that the difference between the gaps is only around $20 \%$. Clearly, the difference between the two procedures comes from the fact that our first-principles-based calculation included all of the five bands crossing the level. However, our main point is that the DOS is spin polarized around the Fermi level, and the superconducting gaps correspond to different spin channels, not different bands.

We note that it is important to consider the effect of magnetic and nonmagnetic impurities on the INT state. Although this is outside the scope of the present Rapid Communication, due to the two full gaps we expect the INT state to be protected from nonmagnetic impurity scattering and a version of the Anderson's theorem [57] to hold.

We showed that an unconventional superconductor in the INT state has at least two gaps, one for each spin flavor, irrespective of the number of Fermi surfaces. Instead of the 
traditional route of ignoring the microscopic complexity of Fermi surfaces, we consider the fully relativistic electronic band structure of $\mathrm{LaNiGa}_{2}$. We perform fully self-consistent computations of its observables by taking a phenomenological pairing model on the Ni atom in the INT state. The pairing model has a single adjustable parameter fixed by the experimental value of $T_{c}$ of the material. There is an excellent agreement between the computed and measured specific heat of the system. We showed that due to the migration of Cooper pairs a subdominant order parameter, magnetization, arises spontaneously, breaking TRS consistent with the zero-field $\mu \mathrm{SR}$ experiment. The salient feature of our calculations is a double-peak structure in the quasiparticle DOS arising from the two spin channels. We have predicted quantitatively the splitting between the two peaks and showed that it is well within the reach of present experimental technology and resolution. We have thus achieved a desired milestone: a quantitative theory of exotic pairing in an unconventional superconductor, namely, $\mathrm{LaNiGa}_{2}$, predicting a smoking-gun signature of its unconventional pairing state.

This research was supported by EPSRC through the project "Unconventional Superconductors: New paradigms for new materials" (grant references EP/P00749X/1 and EP/P007392/1) and the Hungarian National Research, Development and Innovation Office under Contract No. K115632. G.C. also acknowledges support from the European Union's Horizon 2020 research and innovation programme under the Marie Sklodowska-Curie Grant Agreement No. 754510 and thanks Tom G. Saunderson and Hubert Ebert for fruitful discussions. B.U. also acknowledges NKFIH K131938 and BME Nanotechnology FIKP grants. J.Q. thanks Silvia Ramos for useful discussions. We also thank Kazumasa Miyake for extensive discussions.
[1] M. H. Devoret, A. Wallraff, and J. M. Martinis, Superconducting qubits: A short review, arXiv:cond-mat/0411174.

[2] R. L. Fagaly, Superconducting quantum interference device instruments and applications, Rev. Sci. Instrum. 77, 101101 (2006).

[3] R. H. Heffner, J. L. Smith, J. O. Willis, P. Birrer, C. Baines, F. N. Gygax, B. Hitti, E. Lippelt, H. R. Ott, A. Schenck, E. A. Knetsch, J. A. Mydosh, and D. E. MacLaughlin, New Phase Diagram for $(\mathrm{U}, \mathrm{Th}) \mathrm{Be}_{13}$ : A Muon-Spin-Resonance and $H_{c 1}$ Study, Phys. Rev. Lett. 65, 2816 (1990).

[4] G. M. Luke, Y. Fudamoto, K. M. Kojima, M. I. Larkin, J. Merrin, B. Nachumi, Y. J. Uemura, Y. Maeno, Z. Q. Mao, Y. Mori, H. Nakamura, and M. Sigrist, Time-reversal symmetrybreaking superconductivity in $\mathrm{Sr}_{2} \mathrm{RuO}_{4}$, Nature (London) 394, 558 (1998).

[5] G. M. Luke, A. Keren, L. P. Le, W. D. Wu, Y. J. Uemura, D. A. Bonn, L. Taillefer, and J. D. Garrett, Muon Spin Relaxation in $\mathrm{UPt}_{3}$, Phys. Rev. Lett. 71, 1466 (1993).

[6] Y. Aoki, A. Tsuchiya, T. Kanayama, S. R. Saha, H. Sugawara, H. Sato, W. Higemoto, A. Koda, K. Ohishi, K. Nishiyama, and R. Kadono, Time-Reversal Symmetry-Breaking Superconductivity in Heavy-Fermion $\mathrm{PrOs}_{4} \mathrm{Sb}_{12}$ Detected by Muon-Spin Relaxation, Phys. Rev. Lett. 91, 067003 (2003).

[7] L. Shu, W. Higemoto, Y. Aoki, A. D. Hillier, K. Ohishi, K. Ishida, R. Kadono, A. Koda, O. O. Bernal, D. E. MacLaughlin, Y. Tunashima, Y. Yonezawa, S. Sanada, D. Kikuchi, H. Sato, H. Sugawara, T. U. Ito, and M. B. Maple, Suppression of time-reversal symmetry breaking superconductivity in $\operatorname{Pr}\left(\mathrm{Os}_{1-x} \mathrm{Ru}_{x}\right)_{4} \mathrm{Sb}_{12}$ and $\mathrm{Pr}_{1-y} \mathrm{La}_{y} \mathrm{Os}_{4} \mathrm{Sb}_{12}$, Phys. Rev. B 83, 100504(R) (2011).

[8] A. Maisuradze, W. Schnelle, R. Khasanov, R. Gumeniuk, M. Nicklas, H. Rosner, A. Leithe-Jasper, Yu. Grin, A. Amato, and P. Thalmeier, Evidence for time-reversal symmetry breaking in superconducting $\mathrm{PrPt}_{4} \mathrm{Ge}_{12}$, Phys. Rev. B 82, 024524 (2010).

[9] A. D. Hillier, J. Quintanilla, and R. Cywinski, Evidence for Time-Reversal Symmetry Breaking in the Noncentrosymmetric Superconductor $\mathrm{LaNiC}_{2}$, Phys. Rev. Lett. 102, 117007 (2009).

[10] A. D. Hillier, J. Quintanilla, B. Mazidian, J. F. Annett, and R. Cywinski, Nonunitary Triplet Pairing in the Centrosymmetric Superconductor LaNiGa 2 , Phys. Rev. Lett. 109, 097001 (2012).
[11] Z. F. Weng, J. L. Zhang, M. Smidman, T. Shang, J. Quintanilla, J. F. Annett, M. Nicklas, G. M. Pang, L. Jiao, W. B. Jiang, Y. Chen, F. Steglich, and H. Q. Yuan, Two-Gap Superconductivity in $\mathrm{LaNiGa}_{2}$ with Nonunitary Triplet Pairing and Even Parity Gap Symmetry, Phys. Rev. Lett. 117, 027001 (2016).

[12] P. K. Biswas, H. Luetkens, T. Neupert, T. Stürzer, C. Baines, G. Pascua, A. P. Schnyder, M. H. Fischer, J. Goryo, M. R. Lees, H. Maeter, F. Brückner, H.-H. Klauss, M. Nicklas, P. J. Baker, A. D. Hillier, M. Sigrist, A. Amato, and D. Johrendt, Evidence for superconductivity with broken time-reversal symmetry in locally noncentrosymmetric SrPtAs, Phys. Rev. B 87, 180503(R) (2013).

[13] R. P. Singh, A. D. Hillier, B. Mazidian, J. Quintanilla, J. F. Annett, D. McK. Paul, G. Balakrishnan, and M. R. Lees, Detection of Time-Reversal Symmetry Breaking in the Noncentrosymmetric Superconductor $\operatorname{Re}_{6} \mathrm{Zr}$ Using Muon-Spin Spectroscopy, Phys. Rev. Lett. 112, 107002 (2014).

[14] D. Singh, J. A. T. Barker, A. Thamizhavel, D. McK. Paul, A. D. Hillier, and R. P. Singh, Time-reversal symmetry breaking in the noncentrosymmetric superconductor $\mathrm{Re}_{6} \mathrm{Hf}$ : Further evidence for unconventional behavior in the $\alpha$-Mn family of materials, Phys. Rev. B 96, 180501(R) (2017).

[15] T. Shang, G. M. Pang, C. Baines, W. B. Jiang, W. Xie, A. Wang, M. Medarde, E. Pomjakushina, M. Shi, J. Mesot, H. Q. Yuan, and T. Shiroka, Nodeless superconductivity and time-reversal symmetry breaking in the noncentrosymmetric superconductor $\mathrm{Re}_{24} \mathrm{Ti}_{5}$, Phys. Rev. B 97, 020502(R) (2018).

[16] D. Singh, K. P. Sajilesh, J. A. T. Barker, D. McK. Paul, A. D. Hillier, and R. P. Singh, Time reversal symmetry breaking in noncentrosymmetric superconductor $\mathrm{Re}_{6} \mathrm{Ti}$, Phys. Rev. B 97, 100505 (2018).

[17] A. Bhattacharyya, D. T. Adroja, J. Quintanilla, A. D. Hillier, N. Kase, A. M. Strydom, and J. Akimitsu, Broken time-reversal symmetry probed by muon spin relaxation in the caged type superconductor $\mathrm{Lu}_{5} \mathrm{Rh}_{6} \mathrm{Sn}_{18}$, Phys. Rev. B 91, 060503(R) (2015).

[18] J. A. T. Barker, D. Singh, A. Thamizhavel, A. D. Hillier, M. R. Lees, G. Balakrishnan, D. McK. Paul, and R. P. Singh, Unconventional Superconductivity in $\mathrm{La}_{7} \mathrm{Ir}_{3}$ Revealed by Muon Spin Relaxation: Introducing a New Family of Noncentrosymmetric Superconductor that Breaks Time-Reversal Symmetry, Phys. Rev. Lett. 115, 267001 (2015). 
[19] D. Singh, M. S. Scheurer, A. D. Hillier, and R. P. Singh, Timereversal-symmetry breaking and unconventional pairing in the noncentrosymmetric superconductor $\mathrm{La}_{7} \mathrm{Rh}_{3}$ probed by $\mu \mathrm{SR}$, arXiv:1802.01533.

[20] J. Xia, Y. Maeno, P. T. Beyersdorf, M. M. Fejer, and A. Kapitulnik, High Resolution Polar Kerr Effect Measurements of $\mathrm{Sr}_{2} \mathrm{RuO}_{4}$ : Evidence for Broken Time-Reversal Symmetry in the Superconducting State, Phys. Rev. Lett. 97, 167002 (2006).

[21] E. R. Schemm, W. J. Gannon, C. M. Wishne, W. P. Halperin, and A. Kapitulnik, Observation of broken time-reversal symmetry in the heavy-fermion superconductor $\mathrm{UPt}_{3}$, Science 345, 190 (2014).

[22] A. Sumiyama, D. Kawakatsu, J. Gouchi, A. Yamaguchi, G. Motoyama, Y. Hirose, R. Settai, and Y. Ōnuki, Spontaneous magnetization of non-centrosymmetric superconductor $\mathrm{LaNiC}_{2}$, J. Phys. Soc. Jpn. 84, 013702 (2015).

[23] J. F. Annett, Symmetry of the order parameter for high-temperature superconductivity, Adv. Phys. 39, 83 (1990).

[24] M. Sigrist and K. Ueda, Phenomenological theory of unconventional superconductivity, Rev. Mod. Phys. 63, 239 (1991).

[25] J. Quintanilla, A. D. Hillier, J. F. Annett, and R. Cywinski, Relativistic analysis of the pairing symmetry of the noncentrosymmetric superconductor $\mathrm{LaNiC}_{2}$, Phys. Rev. B 82, 174511 (2010)

[26] A. Subedi and D. J. Singh, Electron-phonon superconductivity in noncentrosymmetric $\mathrm{LaNiC}_{2}$ : First-principles calculations, Phys. Rev. B 80, 092506 (2009).

[27] D. J. Singh, Electronic structure and fermiology of superconducting $\mathrm{LaNiGa}_{2}$, Phys. Rev. B 86, 174507 (2012).

[28] J. Chen, L. Jiao, J. L. Zhang, Y. Chen, L. Yang, M. Nicklas, F. Steglich, and H. Q. Yuan, Evidence for two-gap superconductivity in the non-centrosymmetric compound $\mathrm{LaNiC}_{2}$, New J. Phys. 15, 053005 (2013).

[29] J. Chen, L. Jiao, J. L. Zhang, Y. Chen, L. Yang, and H. Q. Yuan, A study of London penetration depth for the noncentrosymmetric superconductor $\mathrm{LaNiC}_{2}$, J. Korean Phys. Soc. 63, 463 (2013).

[30] We stress that in $\mathrm{LaNiGa}_{2}$ a purely nonunitary triplet state is the only possibility compatible with broken TRS due to the low dimensionality of the irreps of the crystal point group.

[31] X. Dai, Z. Fang, Y. Zhou, and Fu-Chun Zhang, Even Parity, Orbital Singlet, and Spin Triplet Pairing for Superconducting $\mathrm{LaFeAsO}_{1-x} \mathrm{~F}_{x}$, Phys. Rev. Lett. 101, 057008 (2008).

[32] T. Nomoto, K. Hattori, and H. Ikeda, Classification of "multipole" superconductivity in multiorbital systems and its implications, Phys. Rev. B 94, 174513 (2016).

[33] P. M. R. Brydon, L. Wang, M. Weinert, and D. F. Agterberg, Pairing of $j=3 / 2$ Fermions in Half-Heusler Superconductors, Phys. Rev. Lett. 116, 177001 (2016).

[34] Y. Yanase, Nonsymmorphic Weyl superconductivity in $\mathrm{UPt}_{3}$ based on $E_{2 u}$ representation, Phys. Rev. B 94, 174502 (2016)

[35] E. M. Nica, R. Yu, and Q. Si, Orbital-selective pairing and superconductivity in iron selenides, npj Quantum Mater. 2, 24 (2017)

[36] D. F. Agterberg, P. M. R. Brydon, and C. Timm, Bogoliubov Fermi Surfaces in Superconductors with Broken Time-Reversal Symmetry, Phys. Rev. Lett. 118, 127001 (2017).
[37] P. M. R. Brydon, D. F. Agterberg, H. Menke, and C. Timm, Bogoliubov Fermi surfaces: General theory, magnetic order, and topology, Phys. Rev. B 98, 224509 (2018).

[38] W. Huang, Y. Zhou, and H. Yao, Exotic Cooper pairing in multiorbital models of $\mathrm{Sr}_{2} \mathrm{RuO}_{4}$, Phys. Rev. B 100, 134506 (2019).

[39] A. Ramires and M. Sigrist, Superconducting order parameter of $\mathrm{Sr}_{2} \mathrm{RuO}_{4}$ : A microscopic perspective, Phys. Rev. B 100, 104501 (2019).

[40] L.-H. Hu and C. Wu, Two-band model for magnetism and superconductivity in nickelates, Phys. Rev. Res. 1, 032046 (2019).

[41] J. L. Lado and M. Sigrist, Detecting nonunitary multiorbital superconductivity with dirac points at finite energies, Phys. Rev. Res. 1, 033107 (2019).

[42] Y. Li and C. Wu, The J-triplet Cooper pairing with magnetic dipolar interactions, Sci. Rep. 2, 392 (2012).

[43] The two-orbital Hubbard model on a square lattice has in general intraorbital Coulomb, interorbital Coulomb, exchange and pair-hopping interactions with symmetry constraints between their respective strengths, offering the possibility of an effective attractive interaction like the one considered here [58]. Nevertheless, we stress that in our approach the constant $U$ is purely phenomenological.

[44] See Supplemental Material at http://link.aps.org/supplemental/ 10.1103/PhysRevB.101.100506 for details of the computational techniques, which includes Refs. [11,27-29,59,60].

[45] K. Miyake, Theory of pairing assisted spin polarization in spin-triplet equal spin pairing: Origin of extra magnetization in $\mathrm{Sr}_{2} \mathrm{RuO}_{4}$ in superconducting state, J. Phys. Soc. Jpn. 83, 053701 (2014).

[46] B. L. Gyorffy, Z. Szotek, W. M. Temmerman, O. K. Andersen, and O. Jepsen, Quasiparticle spectra of high-temperature superconductors, Phys. Rev. B 58, 1025 (1998).

[47] G. Csire, A. Deák, B. Nyári, H. Ebert, J. F. Annett, and B. Újfalussy, Relativistic spin-polarized KKR theory for superconducting heterostructures: Oscillating order parameter in the $\mathrm{Au}$ layer of $\mathrm{Nb} / \mathrm{Au} / \mathrm{Fe}$ trilayers, Phys. Rev. B 97, 024514 (2018).

[48] T. Okabe, Hund's-rule coupling effect in itinerant ferromagnetism, Prog. Theor. Phys. 98, 331 (1997).

[49] A. Georges, L. de Medici, and J. Mravlje, Strong correlations from Hund's coupling, Annu. Rev. Condens. Matter Phys. 4, 137 (2013).

[50] J. E. Han, Spin-triplet $s$-wave local pairing induced by Hund's rule coupling, Phys. Rev. B 70, 054513 (2004).

[51] G. Csire, B. Újfalussy, and J. F. Annett, Nonunitary triplet pairing in the noncentrosymmetric superconductor $\mathrm{LaNiC}_{2}$, Eur. Phys. J. B 91, 217 (2018).

[52] S. Choi, H. J. Choi, J. M. Ok, Y. Lee, W.-J. Jang, A. T. Lee, Y. Kuk, S. B. Lee, A. J. Heinrich, S.-W. Cheong, Y. Bang, S. Johnston, J. S. Kim, and J. Lee, Switching Magnetism and Superconductivity with Spin-Polarized Current in Iron-Based Superconductor, Phys. Rev. Lett. 119, 227001 (2017).

[53] M. Fanciulli, S. Muff, A. P. Weber, and J. H. Dil, Spin polarization in photoemission from the cuprate superconductor $\mathrm{Bi}_{2} \mathrm{Sr}_{2} \mathrm{CaCu}_{2} \mathrm{O}_{8+\delta}$, Phys. Rev. B 95, 245125 (2017).

[54] C. F. Hirjibehedin and Y. Wang, Recent advances in scanning tunneling microscopy and spectroscopy, J. Phys.: Condens. Matter 26, 390301 (2014). 
[55] P. Szabó, P. Samuely, J. Kačmarčík, T. Klein, J. Marcus, D. Fruchart, S. Miraglia, C. Marcenat, and A. G. M. Jansen, Evidence for Two Superconducting Energy Gaps in $\mathrm{MgB}_{2}$ by Point-Contact Spectroscopy, Phys. Rev. Lett. 87, 137005 (2001).

[56] R. Wiesendanger, Spin mapping at the nanoscale and atomic scale, Rev. Mod. Phys. 81, 1495 (2009).

[57] P. W. Anderson, Theory of dirty superconductors, J. Phys. Chem. Solids 11, 26 (1959).
[58] K. Kubo, Pairing symmetry in a two-orbital Hubbard model on a square lattice, Phys. Rev. B 75, 224509 (2007).

[59] P.-G. de Gennes, Superconductivity of Metals and Alloys (CRC Press, Boca Raton, FL, 2018).

[60] V. A. Romaka, Yu. M. Grin, Ya. P. Yarmolyuk, R. V. Skolozdra, and A. A. Yartys, Magnetic and crystallographic characteristics of RNiGa2 compounds (R-rare-earth metal), Ukr. Fiz. Zh. 28, 227 (1983). 